\title{
Long-distance signal transmission cable interface feature extraction and recognition
}

\author{
Xiaobo Wang ${ }^{1, a}$, Yeshuang Tan ${ }^{1, a}$, Huixian Sun ${ }^{1, a} \&$ Shusong Ren ${ }^{2}$ \\ ${ }^{1}$ Ordnance Engineering College, Shijiazhuang, 050003, China \\ ${ }^{2}$ Unit 66208 of PLA, Zhuozou, 072750, China.
}

Keywords: interface signals; feature extraction; recognition.

\begin{abstract}
To improve the long-distance transmission cable interface test automation level, proposed feature extraction based on the type of adaptive signal recognition technology. Through various types of interface signals in time domain and frequency domain characteristics analysis, using interpolation to achieve peak to peak signal accurate judgment, estimated to achieve an effective signal spectrum by Burg spectral estimation algorithm based on AR model. Experiments show that the signal recognition method based on characteristic differences can distinguish different types of long-distance two-wire duplex transmission interface.
\end{abstract}

\section{Introduction}

A certain type of device has variety of external interfaces, manual testing is slow, poor accuracy [1]. Currently, the test interface must know in advance the interface type, choose the appropriate test program. In the case of the use of personnel are not familiar with the device interface, the interface might be a connection error or there is a lot of communication cable, you cannot quickly and accurately identify what type of signal transmission lines which interface connection. Therefore, implement the automation interface recognition technology has been an urgent need. Computer technology and testing technology combined to produce the interface automatic identification technology, test speed, high precision characteristics, can replace the traditional manual interfaces judgment.

To further enhance the level of automation of interface testing by analyzing time domain and frequency domain characteristics feature, set double threshold to achieve adaptive recognition interface signals. Due to the long-distance transmission interface port-based second-tier, Transmission interface compared with the close study of more value in practical applications, In this paper, set single-wire interface cable digital interface (custom here is W interface)、High-rate digital subscriber line interface (HDSL technology system) and single-line interface (G.SHDSL technology system) for high-speed digital line as example, Burg spectral estimation algorithm respectively AR model and spectral peak to peak signal feature extraction by interpolation based, according to statistical decision theory for interface signals classification.

\section{Interface Signal Acquisition}

Interface signal acquisition is the interface signal feature extraction and classification analysis premise. In recent years, with the rapid development of electronic technology and digital signal processing technology, digital processing way has been into the mainstream. Digital acquisition equipment using the interface signal is converted to the corresponding digital signals, and processed for storage. According to Nyquist sampling theorem, digitize the ADC sampling rate must be at least twice the highest frequency signal to avoid aliasing caused by signal distortion. In engineering, considering the performance and the subsequent signal processing components, usually 5-10 times of the need to select the sampling rate.

\section{Signal Feature Extraction and Analysis}

Signal Peak To Peak Extraction. Signal peak to peak signal level refers to the maximum peak and minimum peak difference [2]. Because the interface signals obtained after digitization, is replaced by the value of continuous analog signal by the value of the signal point discrete, signal acquisition 
schematically shown in Figure 1. Although these signals have been sampled analog signal carries all the information, but it is difficult to achieve accurate judgment signal peak to peak. Because the sample time is difficult to achieve alignment signal peak point, there is an error detection result.

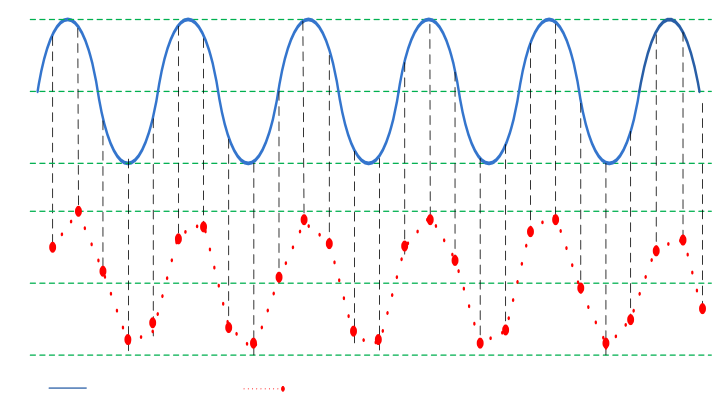

Fig. 1 Signal acquisition schematic

In order to obtain more accurate results, the need for signal processing, commonly used methods of interpolation, fitting, best approximation, numerical integration, fast Fourier transform, solving functional equations and so on. Interpolation method is one of the basic methods of numerical approximation, is determined by the known discrete data interpolation method to obtain a continuous function [3]. It uses a given number of discrete sample points. These sample points are made by continuous. There is a certain smoothness of curves (or surfaces). Interpolation methods used polynomial interpolation (algebraic interpolation), trigonometric interpolation, exponential interpolation, rational interpolation, spline interpolation, etc. By interpolation, in the case may have limited data points, estimated values of the other points, improve the detection accuracy. As shown in Figure 2, the upper portion is a signal waveform obtained after 10 times of the cubic spline interpolation. The lower part is directly sampled signal waveform obtained HDSL interfaces. Before the signal is interpolated maximum $2.92 \mathrm{~V}$, the minimum is $-2.96 \mathrm{~V}$, peak value of $5.88 \mathrm{~V}$. Maximum signal after interpolation is $3.08 \mathrm{~V}$, the minimum is $-3.29 \mathrm{~V}$, peak value of $6.37 \mathrm{~V}$. The interface nominal peak value is $7 \mathrm{~V} \pm 10 \%$, apparently able to obtain more accurate information signal level values by the interpolation method.
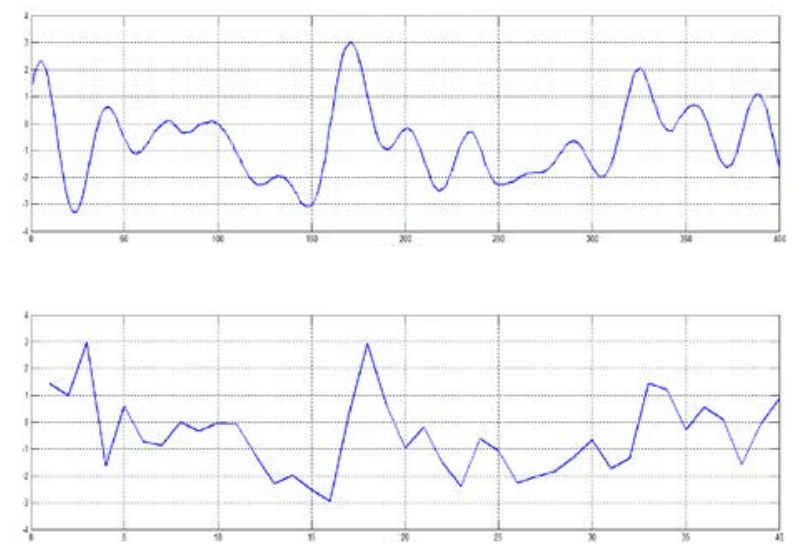

Fig. 2 Signal waveform comparison before and after interpolation

Signal Bandwidth Extraction. Power spectral estimation is a kind of method from a frequency domain analysis of random signals [4], which will help determine the signal occupied bandwidth. The so-called power spectrum estimation is to use the observed data to estimate a number of samples of a stationary random signal power spectrum [5]. Power spectrum estimation is divided into two categories, one kind is the classic spectrum estimation and the other is the model spectrum estimation. Classical spectrum estimation principle is simple, easy to implement. It is estimated that a large variance, spectral resolution is poor. Model in spectral resolution and spectral estimation spectrum compared to the classical method of authenticity has greatly improved. The estimated quality and the appropriateness of the model parameters of the selected model are affected by the degree of improvement.

Model parameter model spectrum estimation in stochastic processes based on the limited data can 
be extracted in the noise of the useful signal from the frequency domain. AR model (autoregressive model) spectrum estimation is the most common method of analysis for spectral analysis of signal characteristics, accurate estimate model parameters need to solve a set of linear equations can be obtained. Burg algorithm is an effective algorithm based on data on the AR model coefficients solving, has been successfully applied in the field of communication filtering, image processing. Burg algorithm based on recursive process on the basis of the data sequence, avoiding the autocorrelation function sequence estimation. Therefore, compared with the autocorrelation method has better frequency resolution. The basic idea is the direct use of linear predictor data observed from the forward and backward prediction of the total sum of the mean square error for the minimum criteria, to estimate the reflection coefficient, and then find the AR model parameter optimization by Levinson recursion formula. It has a good performance in terms of spectral resolution.

Two sinusoidal signals adding noise numbers, for example, seeking power spectrum estimation.

As shown in Figure 3, can be clearly observed Burg algorithm to use AR model power spectrum estimation process is very stable and has a very high resolution.

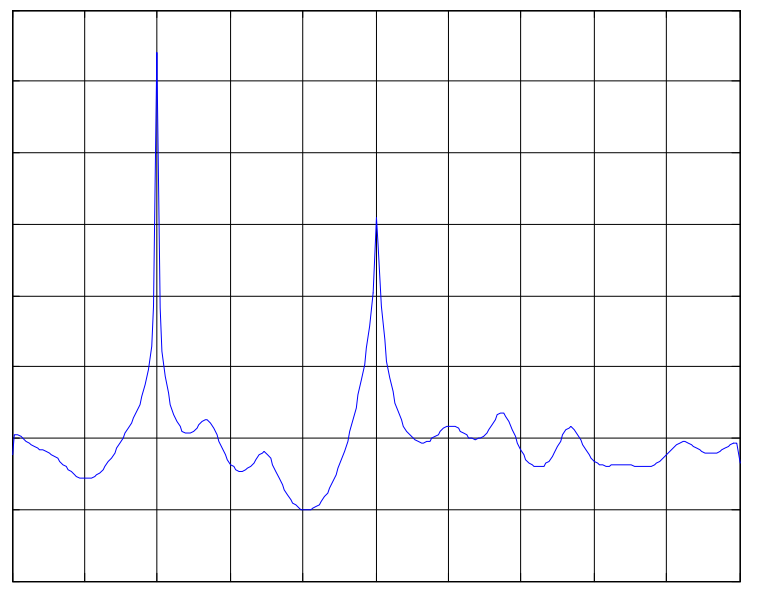

Fig. 3 Burg algorithm based on AR model power spectrum estimation map

Interface signal recognition. Signal recognition is based on observations of its signal characteristics into different categories. Select and determine appropriate recognition method is one of important research content. Signal recognition method is probably divided into three, statistical pattern recognition, decision theory and artificial neural network identification method to identify calculation methods. Decision theory recognizes are used as signal recognition in many engineering applications. The basic idea is through the analysis of the raw data to extract characteristic parameters, directly or indirectly, statistical recognition method choice, produce decision threshold to identify signals. Among them, the decision threshold selection for a great influence on the recognition rate is the core of decision theory recognition method [6]. In order to ensure the minimum bit error rate, usually the optimal decision threshold method.

According to the analysis to obtain time-domain signal characteristic peak to peak voltage and frequency domain characteristics of the band width, decision threshold are set for signal recognition interface. Interface identification flowchart shown in Figure 4, the specific steps: First, by extracting the time-domain characteristic peak to peak voltage, and the decision threshold $\mathrm{V}_{\mathrm{ff}}$ comparison, HDSL interfaces identified. W interfaces and G.SHDSL interface signal voltage peak to peak, respectively $1 \mathrm{~V} 、 2 \mathrm{~V}$, less than HDSL interface signals peak voltage $7 \mathrm{~V}$.Threshold voltage theoretically should choose $[2 \mathrm{~V}, 7 \mathrm{~V}]$, considering various disturbances, using the best decision threshold method chosen decision threshold voltage $4.5 \mathrm{~V}$ as $\mathrm{V}_{\mathrm{ff}}$ in practical applications. When the signal is peak value is greater than the decision threshold $V_{\text {ff }}$ identify HDSL interface. When the signal is peak value is less than the decision threshold $V_{\text {ff }}$ access to the next round of the judgment decision threshold comparison of F. Secondly, by extracting characteristic frequency domain bandwidth, and the decision threshold F compare the difference between $\mathrm{W}$ interfaces and 
G.SHDSL interface signals. W interfaces and G.SHDSL interface signal bandwidth respectively [80 $\mathrm{kHz}-160 \mathrm{kHz}$ ], [0- $850 \mathrm{kHz}$ ]. Frequency-domain signal threshold is set to [160 kHz - $850 \mathrm{kHz}$, considering various disturbances, using the best decision threshold method, select $505 \mathrm{kHz}$ frequency domain as the decision threshold in practical applications F. G.SHDSL interface that is when the band width is greater than the frequency domain decision threshold $\mathrm{F}$, and vice versa for $\mathrm{W}$ interface.

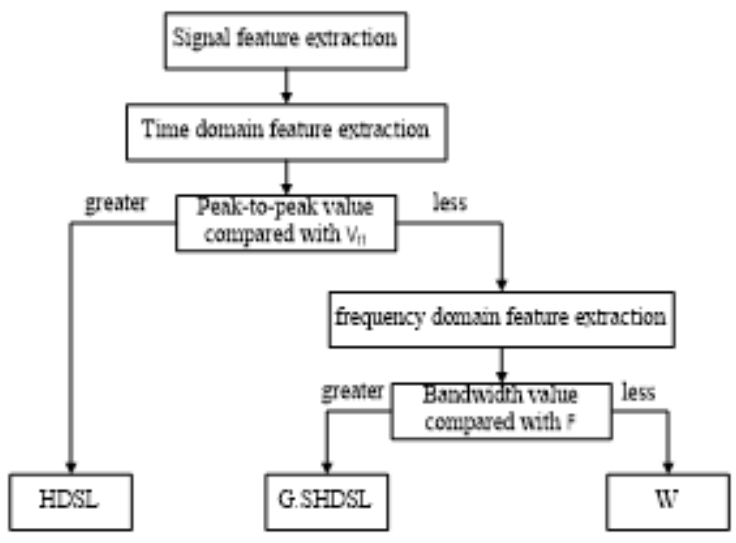

Fig. 4 Interface identification flowchart

\section{Experiments and Conclusions}

By sampling rate, dynamic range, sensitivity, resolution and other parameters and analysis, PXI 8514 data acquisition card of Altai Technologies is used. It has four analog input channels at the same time, the voltage amplitude of each channel of the input signal range is $-5 \sim 5 \mathrm{~V}$. In the digital to analog conversion aspect, it has 14 consecutive A/D converter; maximum sampling rate of $40 \mathrm{Ms} / \mathrm{s}$. Parameter setting data acquisition card direct impact on signal analysis below. The rest include settings set channel, buffer size, trigger control, range scan rate setting and voltage settings. Frequency of the three types of two-wire interface signals up to $850 \mathrm{kHz}$, according to the analysis, the sampling rate of the data acquisition card must be at least $8.5 \mathrm{MHz}$.

Utilizing data acquisition devices, respectively, three types of signal acquisition, the signal obtained after the interpolation process respectively. The peak value is shown as in Table 1. According to the interface signal recognition process by extracting peak to peak with the decision threshold $V_{\text {ff }}$ compare, identify the interface 2 HDSL interfaces. Interface 1 and interface 3 into the next round and compare the decision threshold $\mathrm{F}$.

Table 1 Interface domain characteristic peak to peak

\begin{tabular}{cccc}
\hline Type & Interface 1 & Interface 2 & Interface 3 \\
Peak to peak $/ \mathrm{V}$ & 1.415 & 7.279 & 2.362 \\
\hline
\end{tabular}

The bandwidth of interface 1 and interface 3 were judged by using Burg algorithm based on AR Model. The power spectrum estimation curve is shown in Figure 5. By extracting the band width of the decision threshold $\mathrm{F}$ comparison, it is easy to identify the interface $1 \mathrm{is} \mathrm{W}$ interface, interface 2 is G.SHDSL interface. 

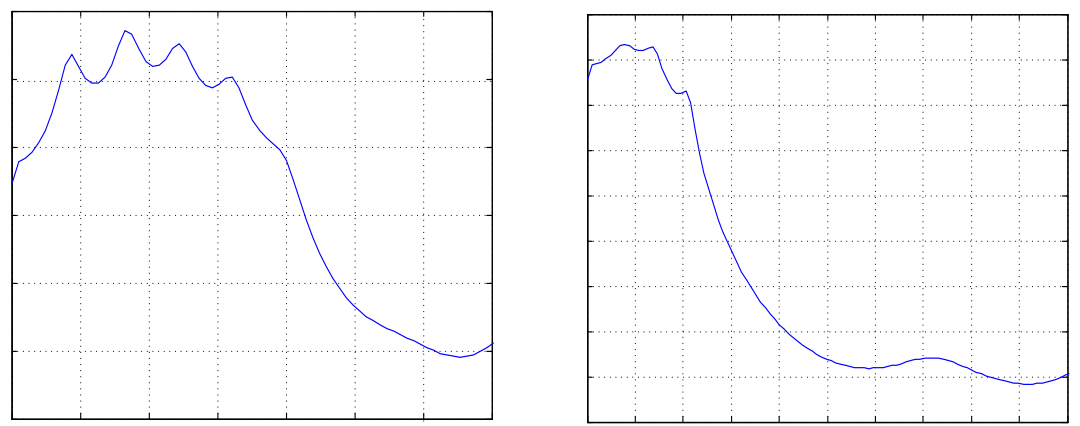

Fig. 5 the power spectrum estimation map of interface 1 and 3

Experiments show that the proposed interface signal characteristic extraction and classification methods, the effective implementation of the W, HDSL and G.SHDSL interfaces effective adaptive recognition categories. Characteristics of the method can be applied to other types of the same twowire interface signal extraction and signal recognition. It provides the basis and prerequisite for long-distance transmission interface tier intelligent testing. It can improve the automated testing level of long-distance transmission cable interface [7], has some practical value in the promotion and application.

\section{References}

[1] Shao Huai Zong, Yuan Xiang Jing, Wu Ying, etc. Design of miniaturized radio communications integrated test system [J]. Ordnance Journal, 2009, 30 (10): 1389-1395.

[2] Du Ting Ke, Yang Junfeng, Song g column, Huang line. A data correlation peak to peak jitter calculation method [J]. University of Science and Technology of China, 2009,39 (6).

[3] Quan Shuang Yan, Cao yang. Application and Research interpolation [J]. Computer and Information Technology. 2007,151 (25): 413-414.

[4] Luo Feng, Duan Pei Pei, WU Shun-jun. Based on a short sequence Burg spectral estimation algorithm[J]. Xi'an University of Electronic Science and Technology: Natural Science, 2005,32 (5): 724-728.

[5] Victor Neagoe, Seeking Pattern Recognition Principles for Intelligent Detection of FSK Signals [A]. 11th IAPR 1992[C].1992,2:721-724.

[6] Huang Chun Lin, Qiu Ling, Shenzhen Kang. Neural network recognition method of digital modulation signals [J]. University of Defense Technology Journal,1999,21(2).

[7] AAAAM R M A. Division - of - amplitude photopolarimeter (DOAP) for the simultaneous measurement of all four Stokes parameters of light. OPTICA ACTA,1982,29(5) : 685-689. 